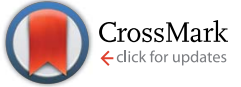

Cite this: RSC Adv., 2017, 7, 12063

Received 3rd January 2017

Accepted 8th February 2017

DOI: $10.1039 / \mathrm{c} 7 \mathrm{ra00055c}$

rsc.li/rsc-advances

\section{Self-healable, tough and highly stretchable hydrophobic association/ionic dual physically cross-linked hydrogels $\uparrow$}

\author{
Yulin Zhang, ${ }^{a}$ Chengxin $\mathrm{Hu}{ }^{a}$ Xu Xiang, ${ }^{\mathrm{b}}$ Yongfu Diao, ${ }^{a}$ Binwei Li, ${ }^{a}$ Linying Shi ${ }^{\mathrm{a}}$ \\ and Rong Ran*a
}

Hydrophobic association (HA) hydrogels have recently attracted much attention since they exhibit high selfhealing ability, remolding capability and shape-memory behavior simultaneously, but their low mechanical strength prevents them from use in many stress-bearing applications. In this work, we describe a novel method for the production of tough and highly stretchable hydrogels with self-healing behavior, tensile strength of 150-300 kPa and stretch at break of 2400-2800\%. Dual physical cross-linking (DPHF) hydrogels were prepared via micellar copolymerization of acrylic acid (AA) and stearyl methacrylate (C18) in an aqueous ferric chloride solution with two different types of surfactant, cetyltrimethylammonium bromide (CTAB) and sodium dodecyl benzene sulfonate (SDBS). The mechanical, rheological, selfhealing and swelling properties of the DPHF hydrogels were investigated and also evaluated as a function of the type of surfactant and the content of ferric ions. The introduction of a moderate content of ferric chloride endowed the hydrogels with excellent strength and self-healing properties simultaneously. Moreover, the structure of the DPHF hydrogels was investigated by IR and SEM analysis. The results were consistent with the results of the mechanical, self-healing and swelling properties tests.

\section{Introduction}

As a soft material containing a large amount of water, hydrogels have been applied in many fields, such as environmental engineering, waste treatment and tissue engineering. ${ }^{1-3}$ Chemically cross-linked hydrogels arise from covalent bond formation, while physically cross-linked hydrogels result from intermolecular interactions such as hydrogen bonds, ${ }^{4,5} \pi-\pi$ stacking, ${ }^{6}$ electrostatic interactions, ${ }^{7,8}$ ionic interactions, chain entanglements, crystallization and other non-covalent interactions., ${ }^{9,10}$ Traditional chemically cross-linked hydrogels tend to display low mechanical strength and poor toughness, due to the inhomogeneity of the chemical crosslinking network and lack of effective energy dissipation under deformation. In order to get better mechanical properties, to date, a good deal of approaches have been proposed in an effort to obtain hydrogels with favourable mechanical properties, such as semi-IPN hydrogels, ${ }^{\mathbf{1 1}}$ slide-ring hydrogels, ${ }^{\mathbf{1 2}}$ hydrophobic association hydrogels (HA gels), ${ }^{13-15}$ double-network (DN) hydrogels, ${ }^{16-18}$ macromolecular microsphere composite (MMC) hydrogels ${ }^{\mathbf{1 9}}$

${ }^{a}$ College of Polymer Science and Engineering, Sichuan University, Chengdu 610065, China.E-mail: ranrong@scu.edu.cn

${ }^{b}$ Chengdu Product Quality Supervision and Inspection Institute, Chengdu 610065, China

$\dagger$ Electronic supplementary information (ESI) available. See DOI: $10.1039 / \mathrm{c} 7 \mathrm{ra} 00055 \mathrm{c}$ and nanocomposite hydrogels. ${ }^{\mathbf{2 0 - 2 2}}$ Among the various hydrogels, DN gels stand out, having gained tremendous attraction due to their remarkable comprehensive mechanical performance. It has been well documented that the majority of DN gels are usually synthesized by two step methods involving two relatively independent networks. However, the covalent bonds serve as cross-links, and once the brittle network is broken the covalent bond cannot be recovered. To overcome this, dynamic physical cross-linking, e.g., ionic bonding or hydrogen bonding, instead of rigid covalent cross-linking in the first network is performed, thereby constructing novel hybrid DN hydrogels. In hybrid DN hydrogels, the covalent bonds are combined with ionic bonds, thus allowing the mechanical properties of the hybrid DN hydrogels to be recovered after internal rupture. ${ }^{23}$ Despite Na-alginate/PAAm DN hydrogels having a high stretchability and toughness, they possess a relatively low fracture strength of $156 \mathrm{kPa}$. When $\mathrm{Ca}^{2+}$ was replaced by other multivalent ions (e.g., $\mathrm{Ba}^{2+}, \mathrm{Al}^{3+}$, and $\mathrm{Fe}^{3+}$ ), the hybrid $\mathrm{DN}$ hydrogels underwent a yielding phenomenon that improved the mechanical properties. ${ }^{24}$ In addition, another approach has been proposed in an effort to obtain this type of hydrogel, namely the dual cross-linked (DC) hydrogel. The existing knowledge of DC hydrogels mainly comes from hybrid physically-chemically cross-linked hydrogels, the networks of which are synchronously cross-linked by covalent bonds and physical interactions, ${ }^{\mathbf{9}, 25}$ which prove to be a fantastic feature in applications. $^{26,27}$ 
HA gels have been proven to possess self-healing and reforming capacities. The hydrophobically associated micelles in HA gels act in a role similar to that of cross-linking agents in chemically cross-linked hydrogels. Generally, HA gels are prepared via micellar copolymerization which combines watersoluble monomers with a small number of other monomers containing hydrophobic groups. As a kind of physically crosslinked hydrogel, the network structure of HA gels is relatively homogeneous and exhibits a desirable energy dissipation mechanism, and results in the remarkable comprehensive mechanical performance; particularly, the elongation could reach more than twenty times the initial length. ${ }^{28}$ The dynamic hydrophobic associations between the hydrophobic domains of polymer chains and surfactant micelles act similarly to the cross-linkers in chemically linked hydrogels. These reversible breakable cross-links are responsible for the rapid self-healing of the hydrogels at room temperature without the need for any stimulus or healing agent. ${ }^{29-31}$ However, the HA gels so far suffer from low mechanical strength which prevents them from use in many stress-bearing applications because of the single cross-linking pattern. In order to increase the mechanical strength of the HA gel, several DN hydrophobic association composite hydrogels have been prepared, by incorporating nanoscopic inorganic materials or crystallinity. These composite hydrogels demonstrated higher mechanical performance and noteworthy adsorption capacity towards different pollutants. However, the self-healing ability of the obtained hydrogels reduced at the same time. ${ }^{26,27}$ It is generally accepted that as the self-healing ability of a hydrogel improves, the mechanical strength decreases. ${ }^{23}$ The challenge of producing self-healable hydrogels with a high mechanical strength is thus inherent. Therefore, up to now the reports about this kind of hydrogel are quite rare. To our knowledge, only Okay et al. ${ }^{\mathbf{1 4}}$ have reported a HA hydrogel with good self-healing behavior and high mechanical performance via micellar copolymerization of AA and stearyl methacrylate (C18) with cetyltrimethylammonium bromide (CTAB) in an aqueous $\mathrm{NaBr}$ solution. Extraction of the free CTAB micelles from the physical gels results in a drastic increase in their comprehensive performance due to the complex formation between PAA and CTAB.

Therefore, this naturally inspires us to explore novel HA based hydrogels, by combining hydrophobic interactions and ionic crosslinking to obtain hybrid DC hydrogels, to simultaneously ameliorate the mechanical strength and healing efficiency. As the most convenient method, introducing multivalent ion salt that is compatible with the PAA HA system seems quite feasible.

Recently, we presented a series of simple strategies for producing a class of physical DN hydrogels such as clay/HA gel, ${ }^{32}$ graphene oxide (GO)/HA gel ${ }^{33}$ and PVA/HA gel. ${ }^{34}$ The obtained gels exhibited good mechanical strength, desirable selfhealing capability and anti-fatigue properties due to incorporating hydrophobic interactions and nanoscopic inorganic materials (or crystallinity). Therefore, on the basis of these results we aimed to manufacture novel physical DC hydrogels composed of hydrophobically associated PAA with physical ionic crosslinking between the ions and the carboxyl groups of PAA.

In this paper, two series of DPHF hydrogels were synthesised using AA as a hydrophilic monomer and C18 as a hydrophobic monomer in an aqueous ferric chloride solution with two kinds of surfactant, anionic surfactant SDBS and cationic surfactant CTAB, respectively. Two DPHF gels without ferric ions were also prepared for comparison using the same procedure.

The mechanical, rheological, self-healing and swelling properties were investigated. The results showed that hydrophobically associated micelles and ion-mediated interactions between the ferric ions and PAA polymer chains were responsible for the enhancement in the mechanical strength of the DPHF hydrogels. Furthermore, the effect of the content of ferric ions and types of surfactant on the rheological, swelling and self-healing properties of the DPHF hydrogels was studied. Finally, the structure of the DPHF hydrogels was investigated by IR and SEM analysis. Herein, we describe a more general approach to prepare DC hydrogels possessing good self-healing and comprehensive mechanical performance, which uses common and easily accessible reagents via one-pot in situ polymerization. Our basic idea is to make use of a DC hydrogel that is made using an established strategy, to bring together two drastically different hydrogels to generate new functionalities or improve properties. Reasonably, combining hydrophobic interactions and ionic crosslinking to obtain hybrid DC hydrogels, to simultaneously ameliorate the mechanical strength and healing efficiency, should be an imperative and instructive strategy. This easy and robust approach can readily be applied to other hydrogels.

\section{Experimental}

\subsection{Materials}

Acrylic acid (AA), iron(III) chloride hexahydrate $\left(\mathrm{FeCl}_{3} \cdot 6 \mathrm{H}_{2} \mathrm{O}\right)$ and potassium persulfate (KPS) were purchased from Tianjin Bodi Chemical Co., Ltd. Sodium dodecyl benzene sulfonate (SDBS) and hexadecyl trimethyl ammonium bromide (CTAB) were provided by Chengdu Kelong Chemical Reagent Factory. Stearyl methylacrylate (C18) was obtained from J\&K Chemical Technology Co., Ltd, and used without further purification.

\subsection{Preparation of hydrogels}

Taking the DPHF gel as an example, AA (20 wt\% in deionized water), SDBS (3 mol\% relative to AA), C18 $(1.5 \mathrm{~mol} \%$ relative to AA) and $\mathrm{FeCl}_{3} \cdot 6 \mathrm{H}_{2} \mathrm{O}(0,0.5 \mathrm{~mol} \%, 1.0 \mathrm{~mol} \%$ and $2.0 \mathrm{~mol} \%$ relative to AA) were added to a $25 \mathrm{ml}$ round-bottomed flask and stirred for 2 hours to form a homogeneous solution. Then, KPS initiator $(0.1 \mathrm{~mol} \%$ relative to $\mathrm{AA})$ was added and the solution was bubbled with nitrogen. Next, the solution was transferred to several narrow-necked glass tubes (10 mm diameter) and the tubes were sealed with an alcohol blast burner. Then, the glass tubes were placed in a water bath at $50{ }^{\circ} \mathrm{C}$ for 20 hours. Finally, the SDBS-DPHF (S-DPHF) gels were obtained. The CTAB-DPHF (C-DPHF) gels were prepared by the same procedure but with the use of CTAB as the surfactant instead of SDBS. Hereafter, 
the S-DPHF gels are labeled as S-DPHF- $x$, and the C-DPHF gels are labeled as C-DPHF- $x$, where $x$ stands for the relative mole fraction of ferric chloride to acrylic acid.

\subsection{Characterization}

Fourier transform infrared (FTIR) spectra of the gels were recorded on a Nicolet 6700 (Thermal Scientific, USA) spectrometer. The morphologies of the gels were examined using a Quanta 250 scanning electron microscope (SEM) (FEI, USA) at $20 \mathrm{kV}$ acceleration voltage. Swelling experiments were performed by completely immersing dried gels into excess deionized water at room temperature. The gels were dried in a vacuum oven at $40{ }^{\circ} \mathrm{C}$ until a constant weight was obtained. The swelling ratio was defined as $S=W_{\mathrm{s}} / W_{\mathrm{d}}-1$, where $W_{\mathrm{s}}$ was the weight in the swollen state and $W_{\mathrm{d}}$ was the weight of the dried sample.

\subsection{Mechanical testing}

Uniaxial and loading-unloading tensile measurements of the hydrogels were taken using an Instron 5965 universal testing system with a $1 \mathrm{kN}$ load cell at a crosshead speed of $100 \mathrm{~mm}$ $\mathrm{min}^{-1}$, and the initial gauge length was $20 \mathrm{~mm}$. Loadingunloading tensile measurements were taken by stretching the hydrogel to a maximum strain of $500 \%$ and then unloading. The energy dissipation capacity was estimated from the area of the hysteresis loop.

Rheology measurements of the hydrogels were taken with a TA AR2000ex rheometer using a parallel plate of diameter 20 $\mathrm{mm}$. The hydrogels were cut into about $5 \mathrm{~mm}$ thick wafers, and a frequency sweep was performed from 0.0159 to $15.9 \mathrm{~Hz}$ at a fixed strain of $1 \%$ at $25{ }^{\circ} \mathrm{C}$.

\subsection{Self-healing experiment}

To prepare self-healing samples, the hydrogels were cut into two parts and the two cut surfaces were kept in contact. The samples were stored in sealed sample bags to prevent hydrogel water loss and the sample bags were placed in a water bath at $25^{\circ} \mathrm{C}$ for an allotted time. To quantify the healing efficiency, the healed samples were subjected to mechanical measurements and compared to the original sample to study the self-healing properties.

\section{Results and discussion}

\subsection{Preparation of the DPHF hydrogels}

Our design rationale for the creation of the DPHF hydrogels is to combine the hydrophobic interactions and ferric ion-mediated ionic interactions between the PAA polymer chains of the hydrogels, as shown in Fig. 1. Two series of DPHF hydrogels were synthesised by one-pot in situ polymerization with the anionic surfactant SDBS and the cationic surfactant CTAB, respectively, in the ferric chloride solution. Two series of DPHF gels without ferric ions were also prepared for comparison using the same procedure. As shown in Fig. 1, both the S-DPHF0 and C-DPHF-0 gels are composed of single hydrophobic association networks. Compared with the S-DPHF-0 gel, the cationic surfactant $\mathrm{CTAB}$ and PAA form a PAA/CTAB complex in the C-DPHF-0 gels, which leads to a more stable and strong hydrophobic association. ${ }^{23,35}$ As ferric ions are introduced into the S-DPHF and C-DPHF gels, dual cross-linked structures form in virtue of the ionic complexation between the ferric ions and the carboxylic acid groups from the PAA chains. Moreover, ferric ions may connect the anionic surfactant SDBS and the carboxylic acid groups to form a more stable hydrophobic association, and further reinforce the S-DPHF gel. On the contrary, ferric ions would destroy the PAA/CTAB complex, leading to the instability of the hydrophobic association and the breakdown of the hydrophobic associated cross-links.

As shown in Fig. 2a, the colors of both the S-DPHF and CDPHF gels are orange. The higher the content of ferric chloride, the darker the gel color is. The S-DPHF gels become hard and brittle with increased ferric chloride content. When the ferric chloride content reached 2 mol\%, the S-DPHF-2 sample became a hard brittle solid. In contrast, the C-DPHF sample was liquid before the ferric chloride content reached $2.5 \mathrm{~mol} \%$ (Fig. S1†). A simple preliminary test for the C-DPHF-0.25 gels is shown in Fig. 2b. The C-DPHF-0.25 gel was stretched, pulled into a film, it supported a $50 \mathrm{~g}$ weight and then the gel lifted a 2 $\mathrm{kg}$ weight. Similarly, the test for the S-DPHF-0.25 gel was done (Fig. S2 $\dagger$ ). It is obvious that the DPHF-0.25 gels exhibited high strength and toughness.

\subsection{Mechanical properties of the DPHF hydrogels}

In order to analyze the mechanical properties of the DPHF hydrogels, stress-strain curves of the DPHF gels were obtained, which are presented in Fig. 3. There is a great difference between the S-DPHF-0 gel and the C-DPHF-0 gel. The S-DPHF gel exhibits good extensibility but poor strength (about $2817 \%$ elongation and $47.8 \mathrm{kPa}$ tensile stress). The repulsive force between SDBS and the carboxylic acid groups of the PAA chains results in unstable hydrophobic associations, therefore the strength of the S-DPHF-0 gel is low. On the contrary, the CDPHF-0 gel exhibits good extensibility and strength (about $1837 \%$ elongation and $248.6 \mathrm{kPa}$ tensile stress), with a drastic increase in its tensile strength due to the complex formation between PAA and CTAB.

The ferric ion concentration plays the key role in the completely different mechanical properties of the two kinds of DPHF gels. There is an improvement in the tensile strength, but the elongation at break of the S-DPHF gel decreases remarkably with the increase of $\mathrm{Fe}^{3+}$ content. It is apparent that the formation of ionic crosslinking between ferric ions and carboxylic acid groups should be responsible for the increase in strength. In addition, the interaction between the $\mathrm{Fe}^{3+}$ and anionic surfactant SDBS leads to more stable hydrophobic association, which further enhances the tensile strength.

Interestingly, the mechanical properties of the C-DPHF gels exhibit a trend of firstly increasing and then decreasing with the increase in ferric content. In the case of a moderate ferric chloride content, the tensile strength and elongation of C-DPHF (e.g. C-DPHF-0.25 has about $2887 \%$ elongation and $308.6 \mathrm{kPa}$ fracture stress) are both higher than those of the C-DPHF-0 gel. 


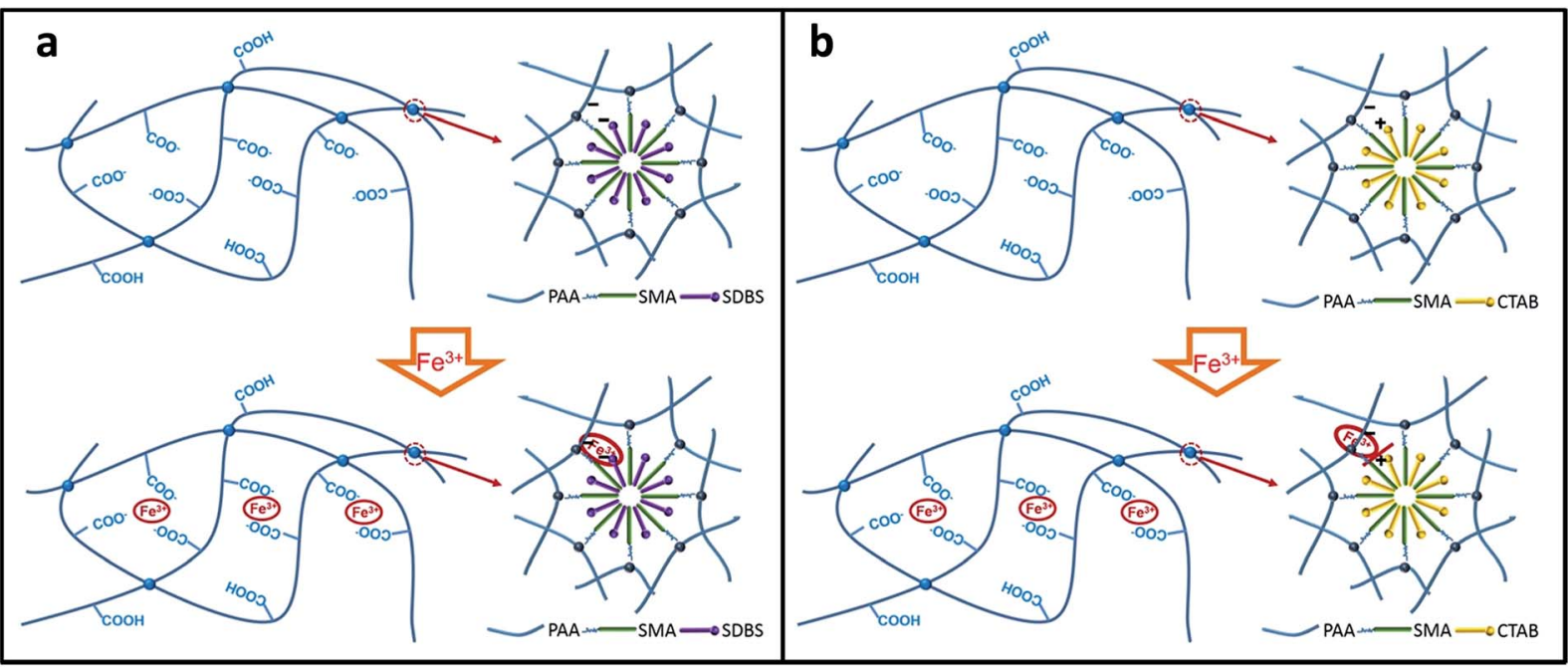

Fig. 1 Schematic illustration of the crosslinking structure: (a) S-DPHF hydrogels; (b) C-DPHF DC gels.

This indicates that at a moderate content, ferric ions could facilitate an increase in the crosslinking density of the gel and the polymerization of PAA, which ensures that more stress can be sustained by the gel sample. ${ }^{26}$ However, a decline of the mechanical properties is observed for the gels with a $\mathrm{Fe}^{3+}$ content higher than $0.5 \mathrm{~mol} \%$. In the case of the C-DPHF gels with a high $\mathrm{Fe}^{3+}$ content, the considerable interactions between $\mathrm{Fe}^{3+}$ and the PAA polymer chains constrained the mobility of the PAA chains to a greater extent, giving rise to a decline in the flexibility of the polymer chains. Therefore, the C-DPHF-0.5 and C-DPHF-1 gels displayed low extensibility with elongations of $1450 \%$ and $470 \%$, respectively. Meanwhile, at a high concentration, $\mathrm{Fe}^{3+}$ could disturb the complex formation between PAA and $\mathrm{CTAB}$, therefore the cross-linking provided by the hydrophobically associated domains weakens, resulting in worsening of the mechanical properties. Moreover, a further increase of the $\mathrm{Fe}^{3+}$ content may retard the radical polymerization and reduce the molecular weight of PAA, and greatly degrade the mechanical properties of the gel. That is, more interactions between $\mathrm{Fe}^{3+}$ and the PAA polymer chains would appear in the DPHF gels, which would mean that more energy would be needed to break the hydrogels. Thereby, the tensile toughness of C-DPHF- 0.25 was also increased in obvious contrast with HA since the tensile toughness was defined as the area underneath the tensile stress-strain curve (Fig. S4†). However, due to the formation of ionic crosslinking between ferric ions and carboxylic acid groups, the toughness of the S-DPHF gels decreased.

Dynamic mechanical measurements were carried out to investigate the rheological properties of the two kinds of DPHF gel. Fig. 4 shows the representative results regarding the viscoelastic properties of the DPHF gels with differing content of ferric ions. Fig. 4a compares the changes in the storage modulus $\left(G^{\prime}\right)$ and the loss modulus $\left(G^{\prime \prime}\right) v s$. frequency at $25^{\circ} \mathrm{C}$, for S-DPHF-0, S-DPHF-0.5 and S-DPHF-1 gels. It was observed from Fig. 4a that $G^{\prime}$ was always higher than $G^{\prime \prime}$ and appeared as almost a plateau over the experimental frequency range. The results showed that all three hydrogels were solid-like, which was evidence of the formation of ionic cross-linked networks in these DPHF gels. Moreover, $G^{\prime}$ was significantly increased with the increase of $\mathrm{Fe}^{3+}$ content. Fig. $4 \mathrm{~b}$ shows that the $G^{\prime}$ values of the C-DPHF gels exhibit a trend of firstly increasing and then

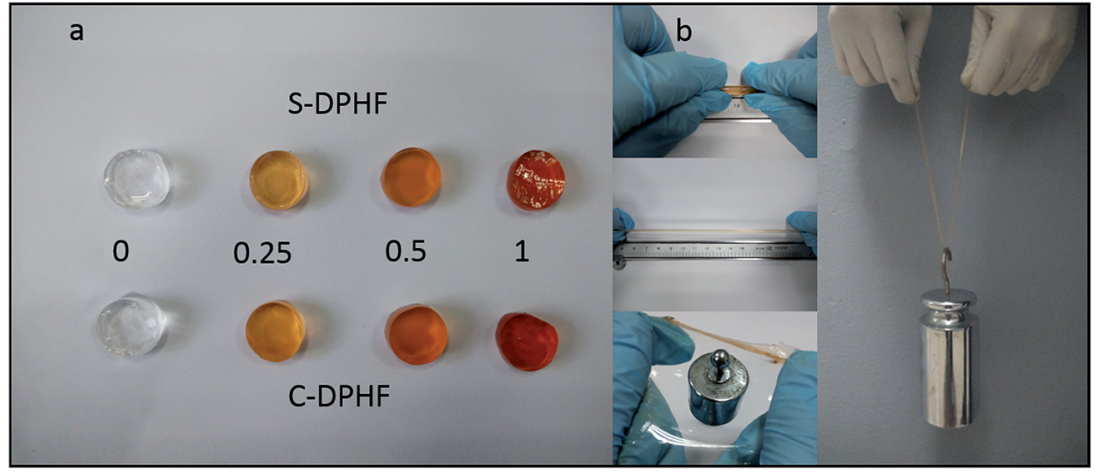

Fig. 2 Photos of (a) the S-DPHF and C-DPHF gels with the ferric chloride content ranging from 0 to 1 mol\%. (b) C-DPHF-0.25 exhibiting excellent mechanical performance: stretching, pulling into film and hanging a weight of $2 \mathrm{~kg}$. 

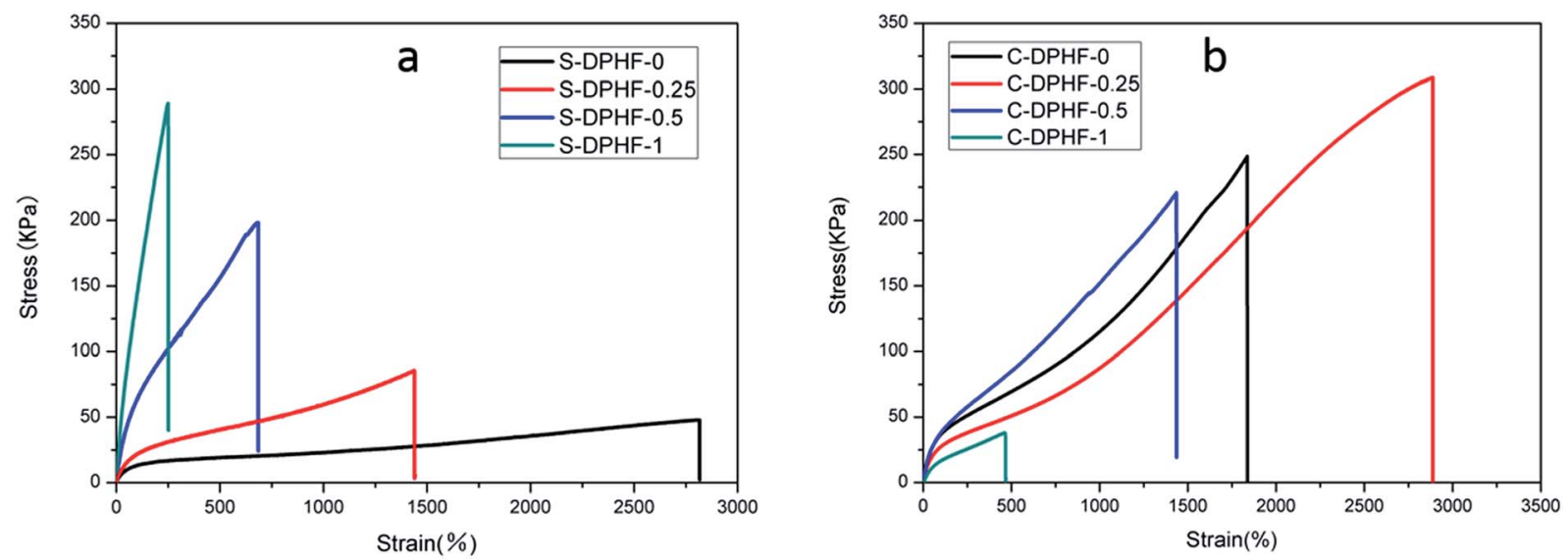

Fig. 3 Stress-strain curves for (a) S-DPHF gels and (b) C-DPHF gels with different ferric chloride content.

decreasing with the increase in ferric content, which is consistent with the result of the mechanical properties test. As mentioned above, the results indicated that the physical $\mathrm{Fe}^{3+}$ cross-linking contributes to the mechanical strength of the DPHF hydrogel, acting cooperatively with the physical hydrophobic association network of PAA.

The energy dissipation capacity was another indicator used to assess the mechanical properties of the hydrogels, and could be acquired from the tensile hysteresis curves during a loadingunloading cycle. The destruction of the physical cross-links such as ionic crosslinking and hydrophobic association is an effective means to dissipate deformation energy. In order to examine the energy dissipation ability of the DPHF gels, we conducted loading-unloading tests at a maximum strain of $500 \%$, with the area between the load and unload stress-strain curves being equal to the energy dissipated. For the C-DPHF-1 and S-DPHF-1 gels, their ultimate elongations were lower than $500 \%$ (Fig. 3). Therefore, these two gels were eliminated to test for comparison in subsequent measurements. The results of the loading-unloading tensile tests of the DPHF gels are shown in Fig. 5.

It is obvious that the S-DPHF-0 gel presents a very small loop, matching a dissipated energy of only $32.1 \mathrm{~kJ} \mathrm{~m}^{-3}$. However, adding only $0.25 \mathrm{~mol} \%$ ferric ions led to a sharp augmentation in the dissipated energy. As for the S-DPHF-0.5 gel, the dissipated energy achieved was even as high as $164.4 \mathrm{~kJ} \mathrm{~m}^{-3}$, more than five times that of the S-DPHF-0 gel (Fig. $5 \mathrm{a}$ and c). It is easy to conclude that the energy is dissipated much more efficiently by the ferric ion mediated crosslinking network of the S-DPHF gel than the S-DPHF-0 gel during the stretching and reverting, thus resulting in higher mechanical strength and toughness. Similar to the trend of the S-DPHF gel, the dissipated energy of the C-DPHF gel increased with the increase of ferric chloride content from 0 to $0.5 \mathrm{~mol} \%$ (Fig. $5 \mathrm{~b}$ and d). However, at $500 \%$ elongation, the tensile strength of the C-DPHF- 0.25 gel was lower than that of the C-DPHF-0 gel. It was tentatively believed that $\mathrm{Fe}^{3+}$ at high concentration could disturb the complex formation between PAA and CTAB, thus degrading the tensile strength of the C-DPHF-0.25 gel.

\subsection{Self-healing properties of the DPHF hydrogels}

The recoverable nature of physical cross-links (non-covalent interactions), e.g. hydrogen bonding, hydrophobic effects, host-guest recognition, and electrostatic and metal-ligand interactions, provides the means for self-repair. A dual
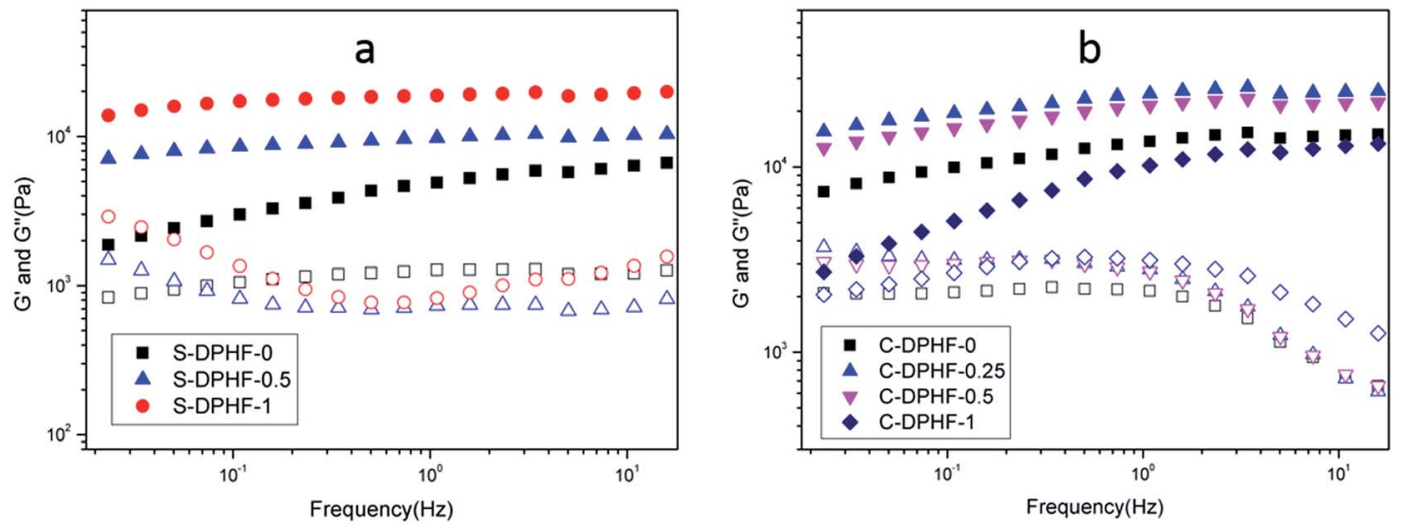

Fig. 4 Frequency dependence of the storage modulus $G^{\prime}$ (solid symbols) and loss modulus $G^{\prime \prime}$ (hollow symbols) at $25^{\circ} \mathrm{C}$ for (a) the $\mathrm{S}$-DPHF gels and (b) the C-DPHF gels. 

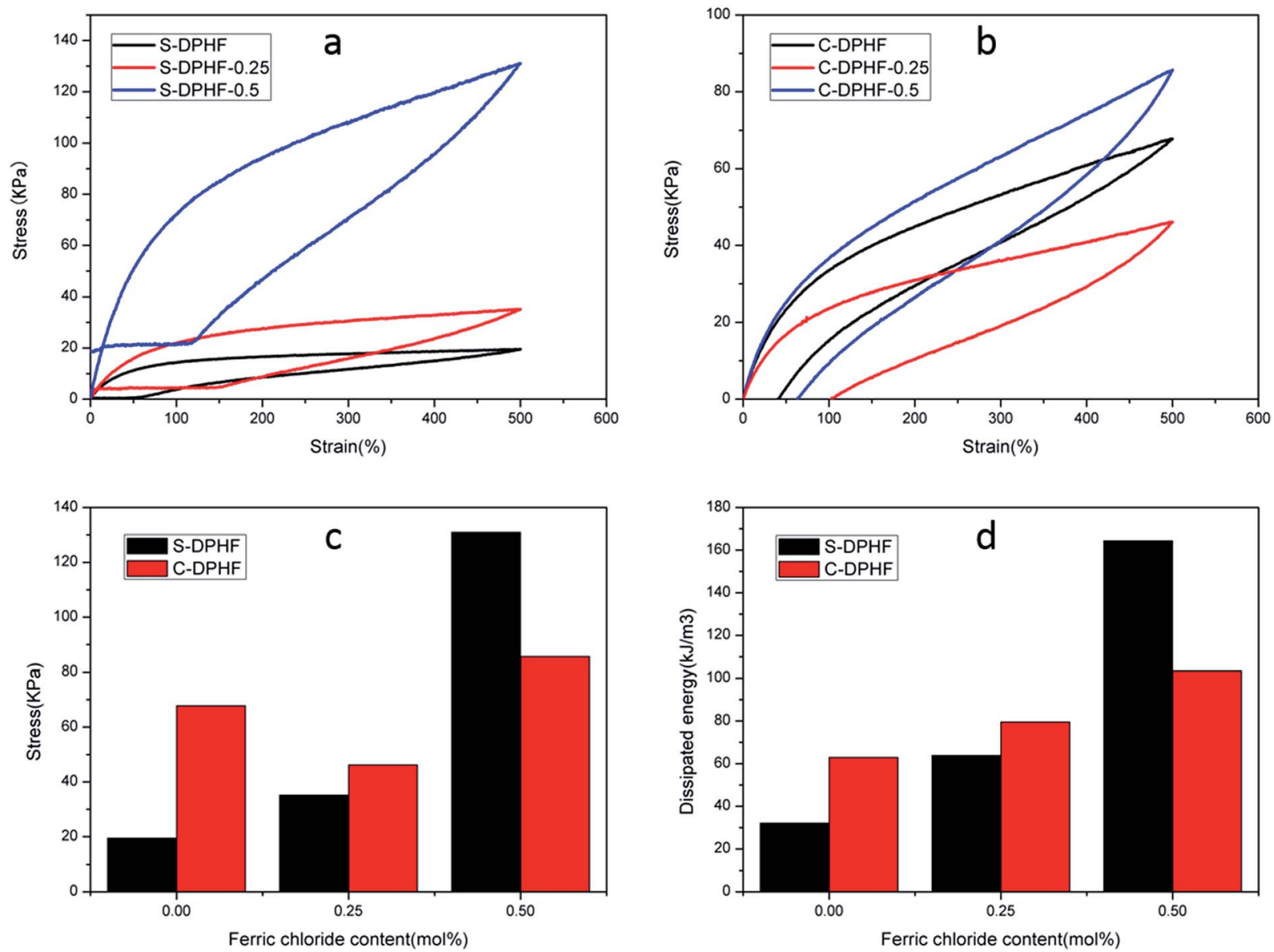

Fig. 5 Loading-unloading tensile curves of the gels at a strain of 500\%: (a) S-DPHF gels and (b) C-DPHF gels. (c) Stress of the S-DPHF and CDPHF gels at a strain of $500 \%$. (d) Dissipated energies of the S-DPHF and C-DPHF gels calculated from the hysteresis loops.

physically cross-linked network was created in the DPHF gels by the introduction of ionic bonding between $\mathrm{Fe}^{3+}$ and carboxylic acid, which endowed the DPHF gels with excellent self-healing properties. In order to evaluate the self-healing properties, we conducted a series of self-healing experiments by tensile testing. We cut all of the self-healing samples into two parts and brought the two cut surfaces together into contact rapidly and preserved them in a wet-keeping cabinet for 2 days at room temperature. Satisfyingly, the healing process of the gels was completely spontaneous, without any auxiliary measures such as heating or adding ferric solvent. The healed samples were tested by taking tensile measurements, and the self-healing efficiency was evaluated by comparing the tensile strengths before and after self-healing, the results of which are shown in Fig. 6 and 7.

Fig. 6 verifies that the healed S-DPHF-0.5 gel achieved a tensile strength of $188 \mathrm{kPa}$, while the healed S-DPHF-0 gel showed a much lower strength of $41.2 \mathrm{kPa}$. By comparing the tensile strengths before and after self-healing, the healing efficiencies of the S-DPHF-0.5, S-DPHF-0.25 and S-DPHF-0 gels were determined as $97 \%, 95 \%$ and $86.2 \%$, respectively (Fig. 6d). The mutual diffusion of the PAA polymer chains, reorganization of the hydrophobically associated domains and the movable
$\mathrm{Fe}^{3+}$ ions at the interface of the two damaged gel samples contribute to the reconstruction of a unique network. As shown in Fig. S3, $\uparrow$ two $5 \mathrm{~mm}$ diameter S-DPHF-0.25 gels were stacked into a cross shape and put into a sample bag. Then, the sample bag was weighted with a water-filled beaker for 20 hours. After the gel was taken out, it could be observed that the strip samples had completely become a whole. The gel was stretched to observe the overlap and no blemishes were found. A $50 \mathrm{~g}$ weight was placed on the overlap with no problem at all. Satisfyingly, the introduction of ferric ions into the S-DPHF system succeeded in increasing the self-healing ability.

In contrast, the self-healing efficiency of the C-DPHF-0 gels (about 73.5\%) was lower compared with that of the S-DPHF0 gels. We further determined the self-healing efficiency of the C-DPHF-0.25 and C-DPHF-0.5 gels, which were $95.8 \%$ and $93.7 \%$, respectively (Fig. $7 \mathrm{~d}$ ).

It is well-established that conventional HA gels are endowed with self-healing capacity due to the structural reorganization of the hydrophobically associated domains. Therefore, in the hydrophobic association/ferric ion dual cross-linked DPHF hydrogels, the ion complexation has a considerable role in promoting the self-healing of the DPHF gels. 

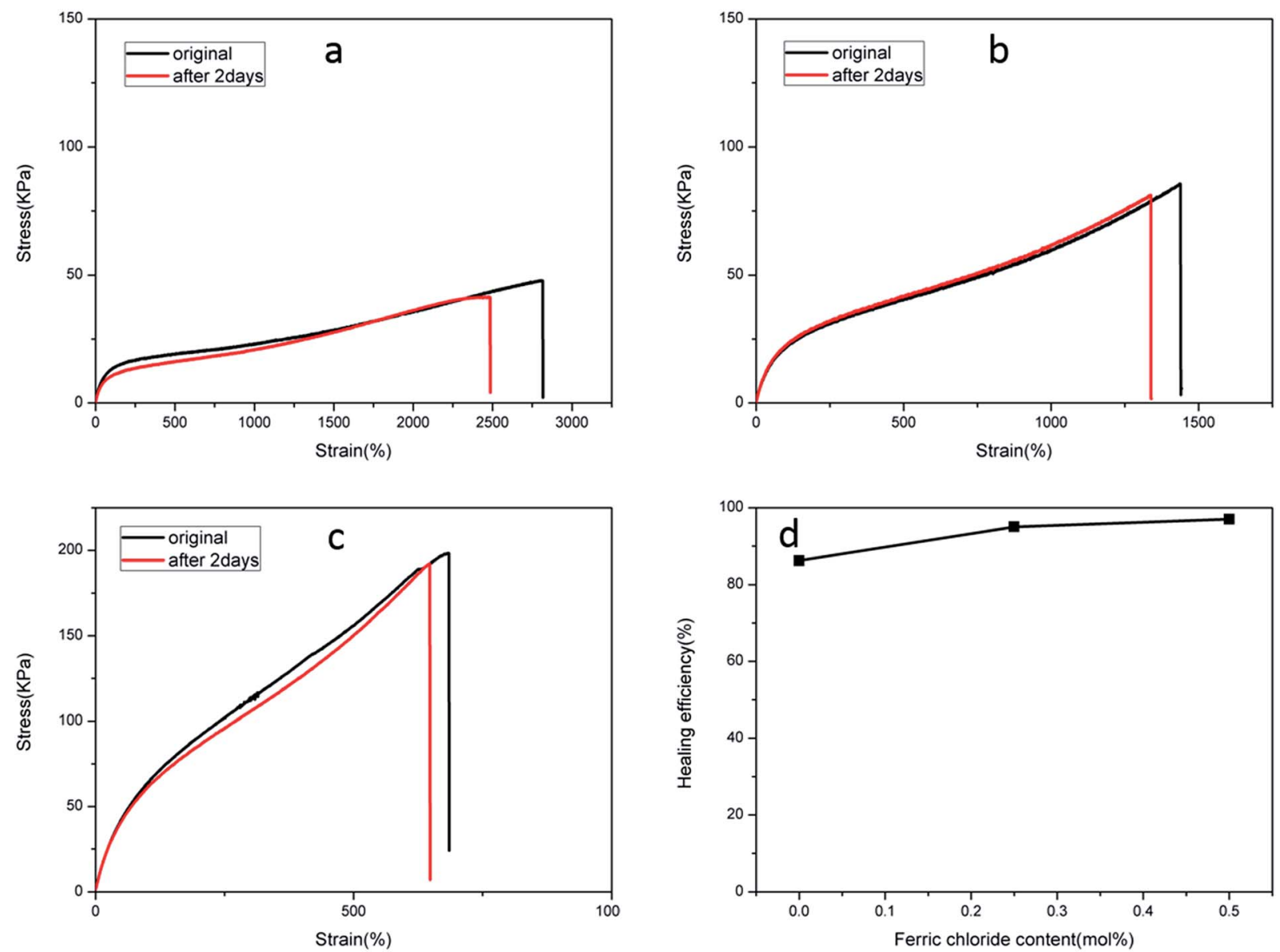

Fig. 6 Tensile stress-stain curves of the healed S-DPHF gels with differing ferric chloride content: (a) S-DPHF-0, (b) S-DPHF-0.25 and (c) SDPHF-0.5. (d) Healing efficiencies of S-DPHF as a function of ferric chloride content.

\subsection{Network structure of the DPHF hydrogels}

The hydrophobically associated cross-linked PAA stabilizes the network structure of the DPHF hydrogels, which is further enhanced by physical cross-linking and ion complexation. It is understandable that the stress cannot be retained at $100 \%$ because such a physical double-network hydrogel is unlikely to have a perfect network structure that prevents any kind of polymer chain slippage or relaxation from happening. Nevertheless, the very low stress relaxation suggests excellent "immobilization" of the polymer chains under deformation, which may ensure that the hydrogel has a good shape fixity and recovery in the shape memory process. In order to verify the proposed structural model (Fig. 1), the swelling behavior of the two kinds of gel was discussed. Fig. 8a shows that the swelling ratio of the S-DPHF gel increased strongly with increasing swelling time and a maximum value was attained in about 1-2 hours. Surprisingly, with increasing swelling time, the swelling ratio exhibits a rapid decrease and then reaches a steady value after a period of time. Because of the osmotic pressure of the SDBS counterions inside the gel network, the gel initially behaves like an ionic gel and thus exhibits a large swelling ratio. However, as SDBS is progressively extracted from the gel network, the osmotic effect disappears and the gel gradually converts into a nonionic gel having a markedly reduced swelling ratio. It is clear that this self-deswelling behavior of the S-DPHF gels should be mainly attributed to the rearrangement of their hydrophobic association structure and ionic interaction. As expected, the swelling ratio of the S-DPHF gels containing a low ferric ion content is always greater than that of the S-DPHF gels containing a high ferric ion content. This phenomenon is consistent with the conclusion that the cross-linking densities for the S-DPHF gels were enhanced with increasing ferric ion content.

The swelling of the C-DPHF gels exhibits a strikingly different behavior (Fig. 8b). In contrast with the S-DPHF-0 gels, the C-DPHF-0 gels deswelled in water, indicating the onset of complexation between PAA and CTAB upon immersion in water. After six days of immersion, the structure of the C-DPHF0 gel was not destroyed. At a certain ferric chloride content, the swelling ratio of the C-DPHF-0.25 gel and the C-DPHF-0.5 gel increased with increasing swelling time and a steady value was attained after a period of time. This observation indicates that a suitable content of ferric chloride leads to the dissociation of the hydrophobic association and PAA/CTAB complex so that the physical crosslinks can dissociate. However, at a higher ferric chloride content, it was found that the C-DPHF-1 gels deswell 

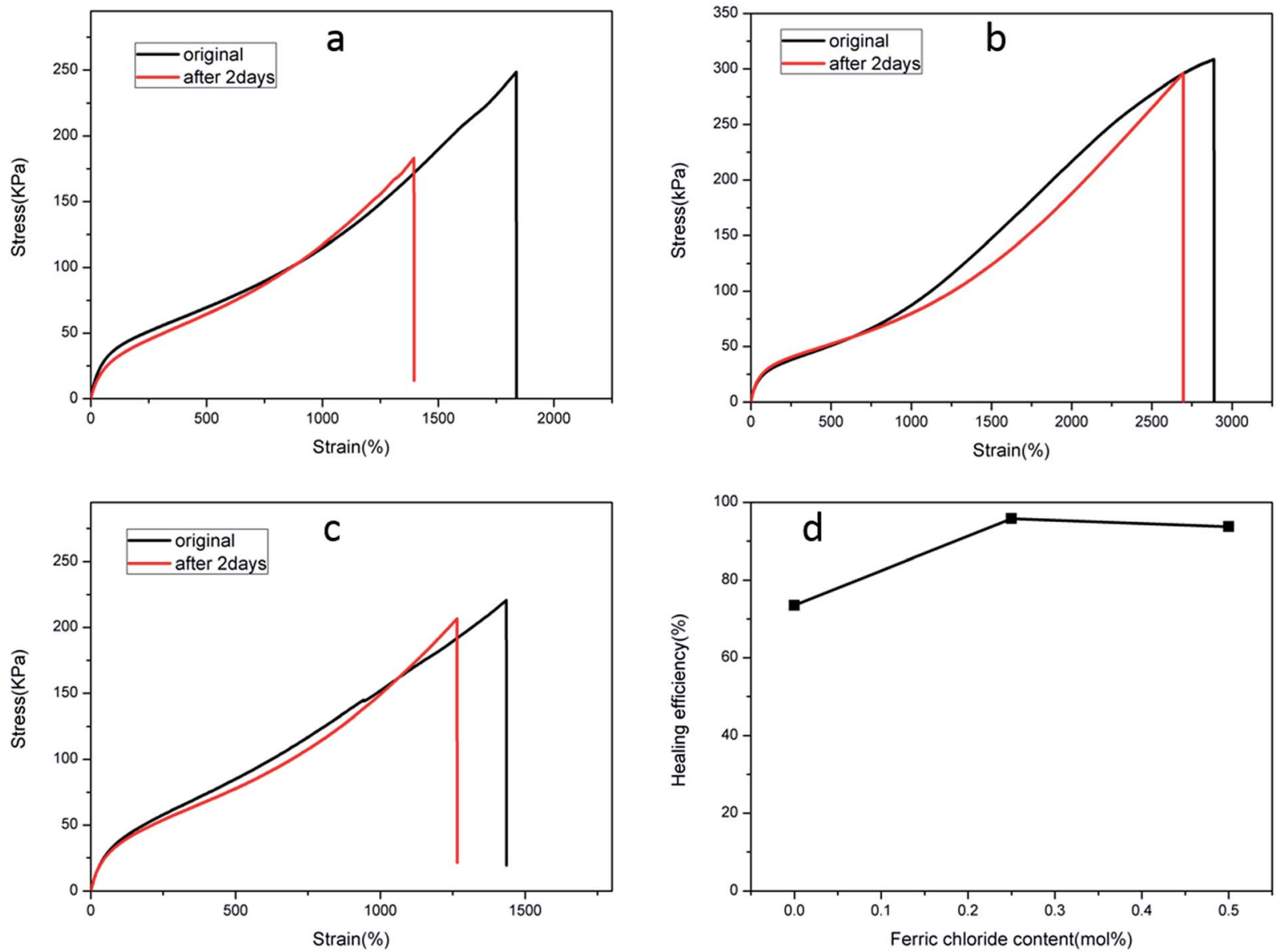

Fig. 7 Tensile stress-stain curves of healed C-DPHF gels with differing ferric chloride content: (a) C-DPHF-0, (b) C-DPHF-0.25 and (c) C-DPHF0.5. (d) Healing efficiencies of the C-DPHF gels as a function of ferric chloride content.

within 3 to 5 days. This indicates that when the ferric chloride content was too large, the PAA/CTAB complex hindered the swelling of the gel.

FTIR spectra of the S-DPHF and C-DPHF gels are presented in Fig. 9. The S-DPHF gel exhibited peaks at $3431 \mathrm{~cm}^{-1}$ for the $-\mathrm{OH}$ group, $2925 \mathrm{~cm}^{-1}$ for the asymmetric stretching vibration of the methylene group, $2857 \mathrm{~cm}^{-1}$ for the symmetric stretching vibration of the methylene group and $1708 \mathrm{~cm}^{-1}$ for the $\mathrm{C}=\mathrm{O}$ stretching of the $-\mathrm{COOH}$ group. The C-DPHF gel exhibited the corresponding peaks at 3431, 2926, 2855 and $1717 \mathrm{~cm}^{-1}$, respectively. These results show that the introduction of ferric chloride has no obvious effect on the FT-IR spectra of the two
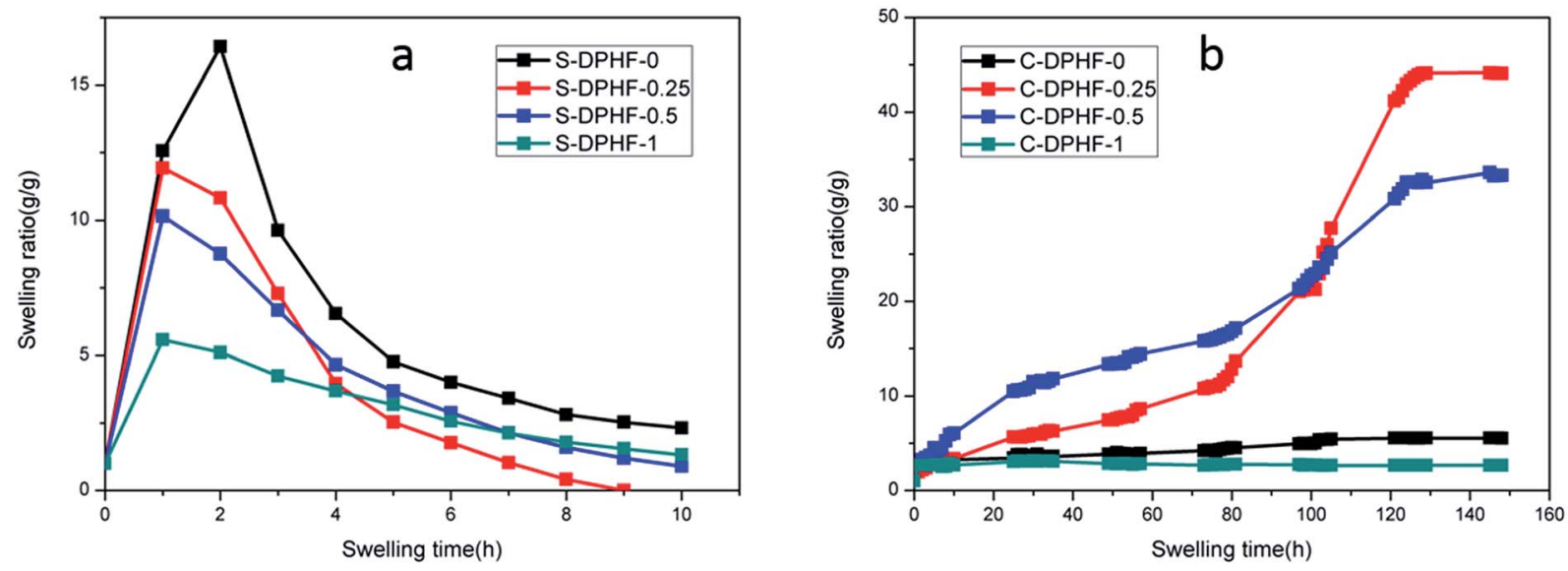

Fig. 8 Time dependence of the swelling ratio for the (a) S-DPHF and (b) C-DPHF gels with differing ferric chloride content. 

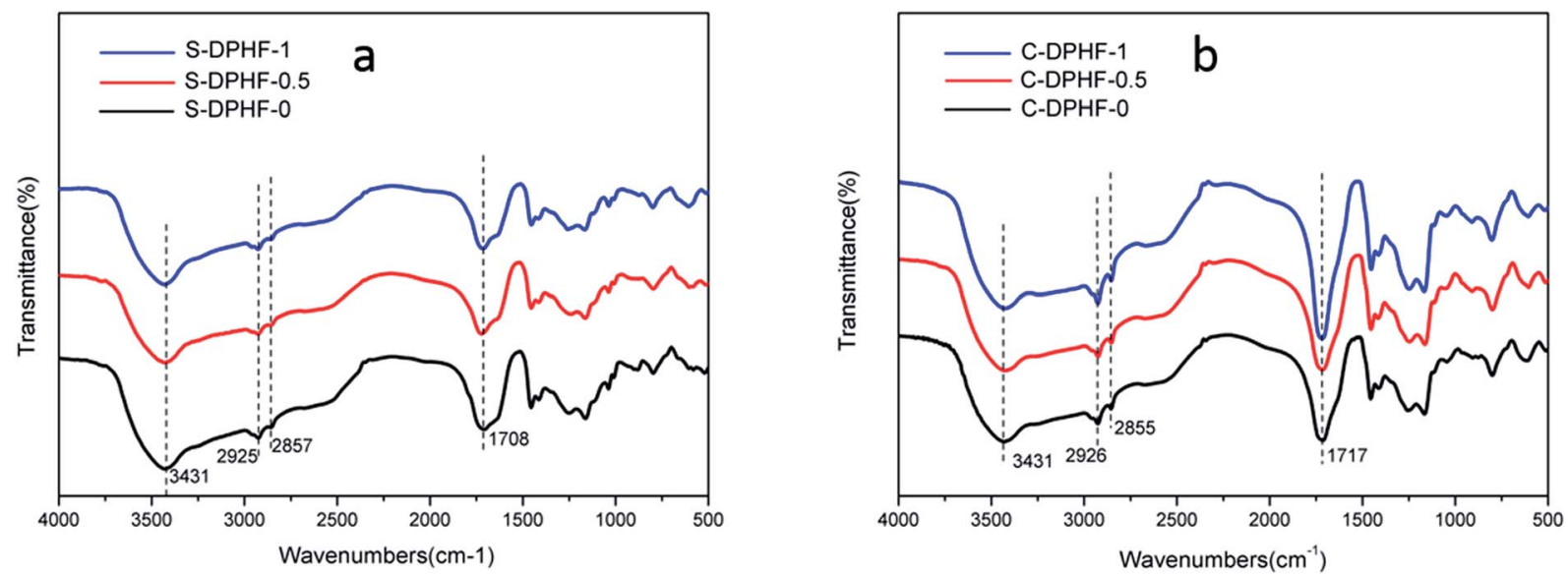

Fig. 9 FTIR spectra of (a) S-DPHF-0, S-DPHF-0.5 and S-DPHF-1 and (b) C-DPHF-0, C-DPHF-0.5 and C-DPHF-1 gels.

kinds of DPHF gel. That is, the FT-IR spectra don't indicate any chemical reaction having taken place.

\subsection{Morphological analysis of the DPHF hydrogels}

As mentioned above, the results of the mechanical and swelling measurements desirably met the viewpoint that the introduction of ferric chloride gave rise to a more compact network structure of the DPHF gels. Thereby, the equilibrium swelling ratio correspondingly changed as ferric chloride was added into the two kinds of gel system. Further intuitive insights into the structural morphology of the two kinds of gel were directly acquired from SEM observations, for which the hydrogel samples were freeze-dried to observe their internal structure. It is clear from Fig. 10 that the internal structures of the S-DPHF gels have changed significantly. Similarly to most lyophilized gels, the inner structure of the S-DPHF-0 gel was characterized by a sponge-like morphology. After the introduction of ferric chloride, the S-DPHF gels showed different morphologies due to the strong interaction between $\mathrm{Fe}^{3+}$ and the PAA chain. Additionally, with the increase of ferric chloride content, the crosslinking density increased significantly. Therefore, the pores gradually became denser and smaller with the increase of the ferric chloride content.

As shown in Fig. 11, it could be seen that the C-DPHF-0 gel presented a much denser homogeneous structure with welldefined pores. Notably, the introduction of ferric ions had a dramatic influence on the size of the pores in the hydrogels. The existence of ferric ions gave rise to a significant decrease in the size of the pores of the C-DPHF- 0.25 gel (Fig. 11b) in clear comparison to that of the C-DPHF-0 gel (Fig. 11a), which indicated that a more compact network structure was generated in the C-DPHF- 0.25 gel by the introduction of ferric ions. Then, when the ferric chloride content was further increased, the pore size of the gels became larger. Correspondingly, the C-DPHF-0.5

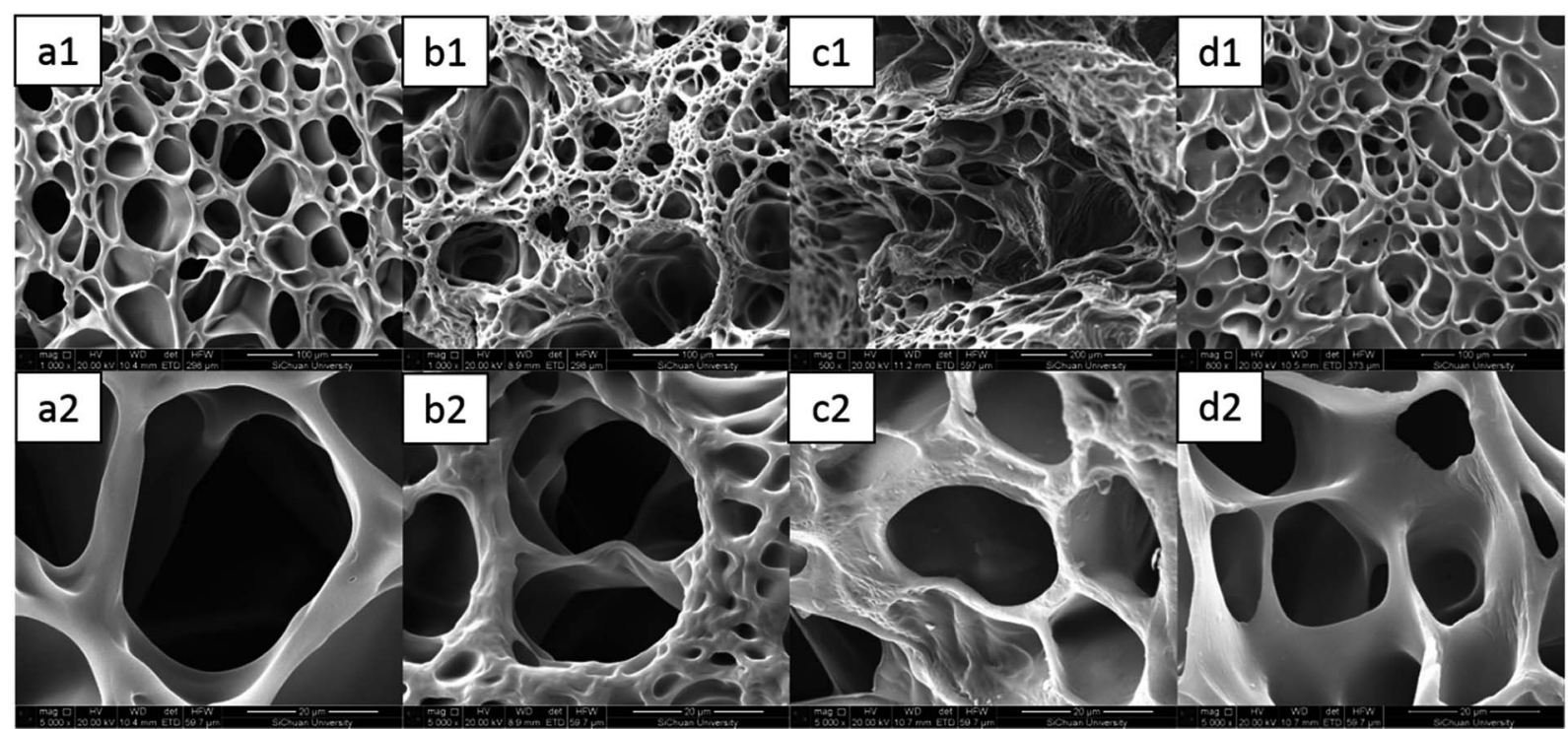

Fig. 10 SEM images of the S-DPHF gels: (a1) S-DPHF-0, (b1) S-DPHF-0.25, (c1) S-DPHF-0.5 and (d1) S-DPHF-1. (a2)-(d2) The gels at high magnification. 


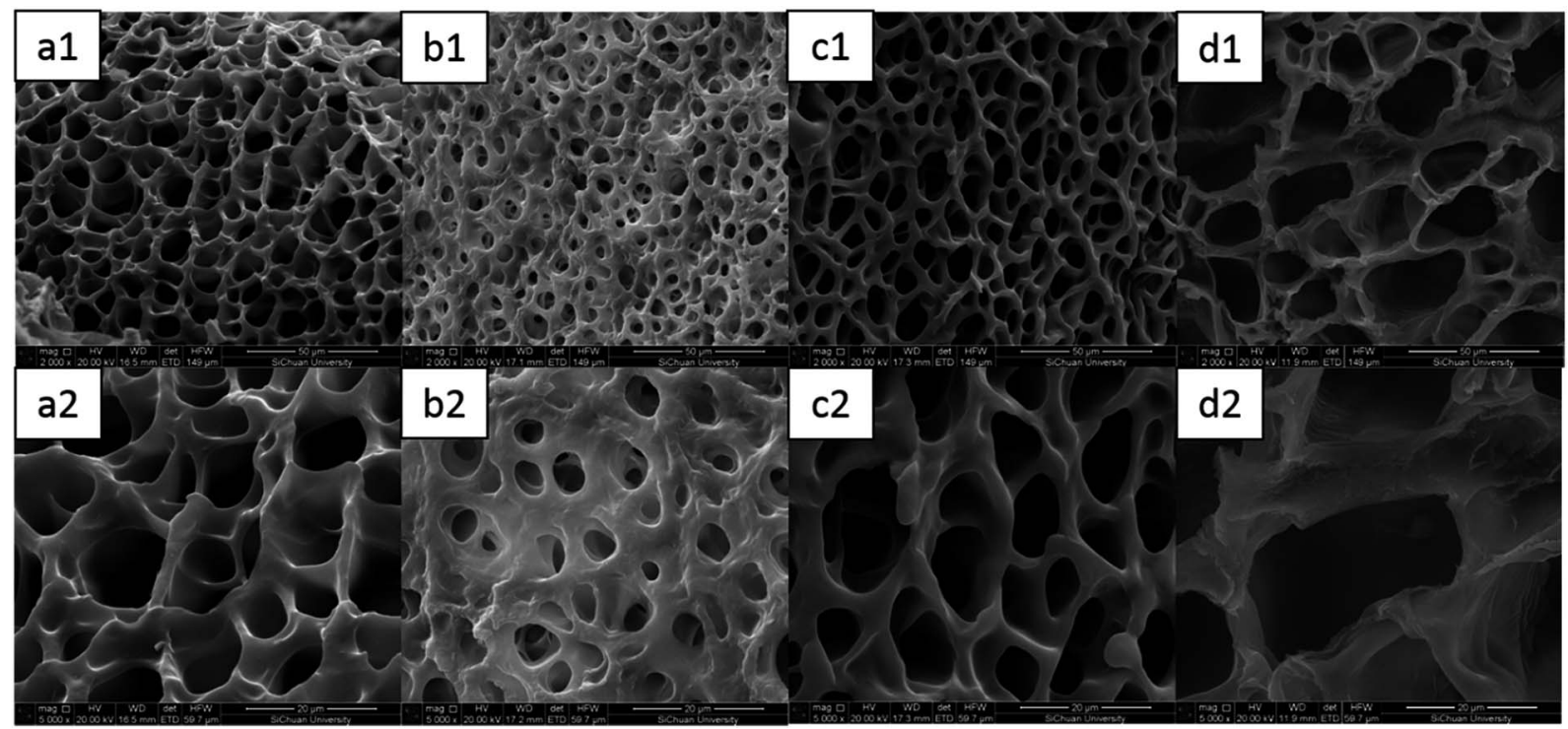

Fig. 11 SEM images of the C-DPHF gels: (a1) C-DPHF-0, (b1) C-DPHF-0.25, (c1) C-DPHF-0.5 and (d1) C-DPHF-1. (a2)-(d2) The gels at high magnification

gel and the C-DPHF-1 gel exhibited larger pore sizes with a loose and irregular pore appearance. It is indicated that the introduction of ferric chloride has two opposite effects on the crosslinking network of the C-DPHF gel. On the one hand, the interaction between the ferric ions and carboxylic acid groups increases the crosslinking density of the hydrogels. On the other hand, ferric ions disturb the interaction between the carboxylic acid groups and CTAB, resulting in the decrease of the crosslinking density. Therefore, the size of the pores of the C-DPHF gels exhibits a trend of firstly decreasing and then increasing with the increase in ferric content. This result is also consistent with the result of the mechanical properties test.

\section{Conclusion}

In summary, hydrophobically associated PAA hydrogels were combined with ferric chloride to obtain a new kind of DPHF hydrogel. Two series of DPHF hydrogels have been successfully prepared via micellar copolymerization of acrylic acid and stearyl methacrylate (C18) in an aqueous ferric chloride solution with two different types of surfactant, CTAB and SDBS, respectively. The mechanical, rheological, self-healing and swelling properties of the DPHF hydrogels were investigated and also evaluated as a function of the type of surfactant and the content of ferric ions. Generally, the mechanical properties and healing efficiencies of the S-DPHF gel were enhanced with the increase of the ferric chloride content, however the swelling ratio decreased with the increase of the ferric chloride content. In clear contrast with the S-DPHF gels, the mechanical properties, healing efficiencies and swelling ratio of the C-DPHF gels exhibit a trend of firstly increasing and then decreasing with the increase in ferric content. Moreover, the structure of the DPHF hydrogels was investigated by IR and SEM analysis. The results were consistent with the result of the mechanical, self-healing and swelling properties test. It could be concluded that the ion complexation has a considerable role in promoting the selfhealing properties of the two kinds of DPHF hydrogel. In addition, hydrophobically associated micelles and ferric ionmediated ionic interactions between the PAA polymer chains were responsible for the enhancement in the mechanical strength of the DPHF hydrogels. The introduction of a moderate content of ferric chloride endowed the hydrogels with excellent strength and self-healing properties. As mentioned above, combining these excellent properties, this kind of hydrogel possesses a wide range of potential applications, such as in automatic control systems and soft robotics. The experimental results also provide some reference for the preparation of high strength HA/ferric chloride composite hydrogels. Our work provides an easy and robust approach for combining hydrophobic interactions and ionic crosslinking to obtain hybrid DC hydrogels with good mechanical strength and self-healing efficiency.

\section{Acknowledgements}

This work was supported by the National Natural Science Foundation of China (Grant 51173121, 51403132), Sichuan Ministry of Science, Technology Project (Grant 2015GZX0226, 2016GZ0300), Innovation Team Program of Science \& Technology Department of Sichuan Province (Grant 2014TD0002) and Cooperation strategic projects of the Luzhou governments and Sichuan University (Grant 2015CDLZ-G13).

\section{References}

1 J. B. Leach and C. E. Schmidt, Biomaterials, 2005, 26, 125135.

2 S. V. Vlierberghe, P. Dubruel and E. Schacht, Biomacromolecules, 2011, 12, 1387-1408.

3 H. Li, J. Bai, Z. Shi and J. Yin, Polymer, 2016, 85, 106-113. 
4 S. Jin, M. Liu, F. Zhang, S. Chen and A. Niu, Polymer, 2006, 47, 1526-1532.

5 D. Han, X. Tong, O. Boissière and Y. Zhao, ACS Macro Lett., 2012, 1, 57-61.

6 Y. Xu, Q. Wu, Y. Sun, H. Bai and G. Shi, ACS Nano, 2010, 4, 7358-7362.

7 A. B. South and L. A. Lyon, Angew. Chem., Int. Ed., 2010, 49, 767-771.

8 Q. Wang, J. L. Mynar, M. Yoshida, E. Lee, M. Lee, K. Okuro, K. Kinbara and T. Aida, Nature, 2010, 463, 339-343.

9 D. C. Tuncaboylu, A. Argun, M. P. Algi and O. Okay, Polymer, 2013, 54, 6381-6388.

10 Q. Yang, C. Song, Q. Chen, P. Zhang and P. Wang, J. Polym. Sci., Part B: Polym. Phys., 2008, 46, 2465-2474.

11 W. Wang and A. Wang, Carbohydr. Polym., 2010, 80, 10281036.

12 T. Karino, Y. Okumura, C. Zhao, T. Kataoka, K. Ito and M. Shibayama, Macromolecules, 2005, 38, 6161-6167.

13 G. Jiang, C. Liu, X. Liu, Q. Chen, G. Zhang, M. Yang and F. Liu, Polymer, 2010, 51, 1507-1515.

14 V. Can, Z. Kochovski, V. Reiter, N. Severin, M. Siebenbürger, B. Kent, J. Just, J. P. Rabe, M. Ballauff and O. Okay, Macromolecules, 2016, 49, 7442-7449.

15 I. Jeon, J. Cui, W. R. K. Illeperuma, J. Aizenberg and J. J. Vlassak, Adv. Mater., 2016, 28, 4678-4683.

16 J. P. Gong, Y. Katsuyama, T. Kurokawa and Y. Osada, Adv. Mater., 2003, 15, 1155-1158.

17 M. Rikkoukalourkoti, E. N. Kitiri, C. S. Patrickios, E. Leontidis, M. Constantinou, G. Constantinides, X. Zhang and C. M. Papadakis, Macromolecules, 2016, 49, 1731-1742.

18 Y. Huang, M. Zhong, Y. Huang, M. Zhu, Z. Pei, Z. Wang, Q. Xue, X. Xie and C. Zhi, Nat. Commun., 2015, 6, 10310.

19 T. Huang, H. G. Xu, K. X. Jiao, L. P. Zhu, H. R. Brown and H. L. Wang, Adv. Mater., 2007, 19, 1622-1626.
20 K. Haraguchi, R. Farnworth, A. Akira Ohbayashi and T. Takehisa, Macromolecules, 2003, 36, 5732-5741.

21 G. Sun, Z. Li, R. Liang, L.-T. Weng and L. Zhang, Nat. Commun., 2016, 7, 12095.

22 K. Haraguchi, H. J. Li, K. Matsuda, T. Takehisa and E. Elliott, Macromolecules, 2005, 38, 33-41.

23 J. Y. Sun, X. Zhao, W. R. K. Illeperuma, O. Chaudhuri, K. H. Oh, D. J. Mooney, J. J. Vlassak and Z. Suo, Nature, 2012, 489, 133-136.

24 C. H. Yang, M. X. Wang, H. Haider, J. H. Yang, J. Y. Sun, Y. M. Chen, J. Zhou and Z. Suo, ACS Appl. Mater. Interfaces, 2013, 5, 10418-10422.

25 B. H. L. S. A. Robb, R. McLemore and B. L. Vernon, Biomacromolecules, 2007, 8, 2294-2300.

26 B. Yang, Y. Zhang, X. Zhang, L. Tao, S. Li and Y. Wei, Polym. Chem., 2012, 3, 3235-3238.

27 J. Cui and C. A. Del, Chem. Commun., 2012, 48, 9302-9304.

28 J. Chen, Y. Ao, T. Lin, X. Yang, J. Peng, W. Huang, J. Li and M. Zhai, Polymer, 2016, 87, 73-80.

29 D. C. Tuncaboylu, M. Sari, W. Oppermann and O. Okay, Macromolecules, 2011, 44, 4997-5005.

30 G. Jiang, C. Liu, X. Liu, G. Zhang, M. Yang, Q. Chen and F. Liu, J. Macromol. Sci., Part A: Pure Appl.Chem., 2010, 47, 335-342.

31 A. B. Ihsan, T. L. Sun, T. Kurokawa, S. N. Karobi, T. Nakajima, T. Nonoyama, C. K. Roy, F. Luo and J. P. Gong, Macromolecules, 2016, 49, 4245-4252.

32 W. Cui, Z. J. Zhang, H. Li, L. M. Zhu, H. Liu and R. Ran, RSC Adv., 2015, 5, 52966-52977.

33 W. Cui, J. Ji, Y. F. Cai, H. Li and R. Ran, J. Mater. Chem. A, 2015, 3, 17445-17458.

34 Y. Zhang, M. Song, Y. Diao, B. Li, L. Shi and R. Ran, RSC Adv., 2016, 6, 112468-112476.

35 M. Zhong, X. Y. Liu, F. K. Shi, L. Q. Zhang, X. P. Wang, A. G. Cheetham, H. Cui and X. M. Xie, Soft Matter, 2015, 11, 4235-4241. 